\title{
Zur besonderen Problematik von Kontaktallergien bei Instrumentalisten*
}

\author{
Contact Allergies in Musicians
}

Autoren

Institut

\section{E. R. Gasenzer, E. A. M. Neugebauer}

IFOM - Institut für Forschung in der Operativen Medizin, Department für Humanmedizin, Universität Witten/Herdecke
Bibliografie

DOI http://dx.doi.org/

10.1055/s-0032-1326289

Akt Dermatol 2013; 39: 389-396

(c) Georg Thieme Verlag KG

Stuttgart · New York

ISSN 0340-2541

\section{Korrespondenzadresse} Dr. Elena Romana Gasenzer IFOM - Institut für Forschung in der Operativen Medizin, Department für Humanmedizin, Universität Witten/Herdecke Ostmerheimer Str. 200,

Haus 38

51109 Köln

elena-romana.gasenzer@ gmx.de

\section{Zusammenfassung \\ $\nabla$}

In den letzten Jahren nimmt die Zahl der allergischen Erkrankungen aller Art stetig zu. Dabei handelt es sich um fehlgeleitete Abwehrreaktionen des Immunsystems. Bei Musikern ist das Krankheitsbild der allergischen Kontaktdermatitis ein häufig auftretendes Problem. Auslöser der allergischen Reaktion sind bei ihnen oftmals die im Musikinstrumentenbau verwendeten tropischen Holzarten. Rosenholzgewächse wie Ebenholz oder Palisander enthalten in hohem Maß Neoflavonoide, die bei lang andauerndem Hautkontakt eine Allergie auslösen können. In der Holzverarbeitung und im Musikinstrumentenbau tritt dementsprechend durch Einatmen der im Holzstaub enthaltenen Stoffe die sog. Holzstauballergie auf. Besonders Holzbläser, hohe Streicher und Perkussionisten sind gefährdet, eine Kontaktdermatitis zu entwickeln: Holzblasinstrumente sowie die Schlägel von Schlaginstrumenten wer-

\section{Einleitung}

\section{$\nabla$}

Allergien sind übertriebene Abwehrreaktionen des Immunsystems aufgrund einer Konfrontation mit sonst harmlosen Umweltstoffen, die sich in Atemwegen, an Schleimhäuten, an der Haut oder auch im Gastrointestinaltrakt abspielen. Entsprechend zeigen sich Symptome wie Schleimhautschwellungen, Asthma-Anfälle, Hautreaktionen oder Durchfälle. Im schlimmsten Fall kommt es zu einem lebensbedrohlichen „allergischen Schock“, der als akuter Notfall zu behandeln ist. Ausgelöst wird die allergische Reaktion durch Allergene. Darunter versteht man Antigene, gegen die sich die Immunantwort richtet. Je nach Art der Allergenquelle (Pollen, Tierhaare, Nahrungsmittel) handelt es sich dabei in der Regel

* Erstpublikation in Dtsch Med Wochenschr 2012; 137: den aufgrund der Klangeigenschaften tropischer Hölzer vorwiegend aus Ebenholz oder Palisanderarten gefertigt. Weil inzwischen hochwertige Instrumente aus tropischen Hölzern im allgemeinen Musikinstrumentenhandel verfügbar sind, können auch Laien und Hobby-Musiker an einer Kontaktallergie erkranken. Bei Querflöten und Blechblasinstrumenten besteht die Gefahr, eine allergische Reaktion gegen das im Metall enthaltende Nickel oder Messing zu entwickeln. Besonders kritisch stellt sich dieses Problem bei Kindern dar, insbesondere, wenn sie bereits eine Allergie - etwa auf Pollen oder Lebensmittel haben. Eltern und Musiklehrer wissen meistens nicht, welche Materialen im Instrument ihres Kindes stecken. Hier ist auch der Arzt gefragt, seine Patienten bzw. deren Eltern entsprechend $\mathrm{zu}$ beraten und ggf. beim Vorhandensein von Ekzemen an Händen, Mund oder Gesicht auf mögliche Ursachen anzusprechen.

um Proteine. Aufgrund der fehlgeleiteten Reaktion des humoralen Immunsystems kommt es nach dem Kontakt mit einem Antigen zur Bildung von IgE-Antikörpern gegen das eigentlich harmlose Antigen, das nun ein Allergen ist: Es werden Allergen-spezifische IgE-Antikörper gebildet [34]. Die in den Körper gelangten Allergene werden von den dendritischen Zellen des Immunsystems aufgenommen und prozessiert. Diese tragen spezielle Rezeptoren auf ihrer Zelloberfläche, deren Aufgabe darin besteht, sog. naive T-Zellen-Allergenbruchstücke zu repräsentieren [23]. Erst durch diese Repräsentation werden die naiven T-Zellen zu aktivierten Th2-Zellen. Auch naive B-Zellen treten in Kontakt mit dem Allergen. Treffen diese B-Zellen dann auf aktivierte Th2-Zellen, interagieren beide Zell-Typen: Die B-Zelle kann jetzt ein Allergen-spezifisches Immunglobulin E (IgE) bilden und setzt dieses in großen Mengen frei. Das IgE wird von anderen Zellen über ihre Rezeptoren 
an der Zelloberfläche erkannt, die dann ihrerseits auf den „bösen Eindringling" reagieren [54]. Pathophysiologisch sind vier Typen (I-IV) von Allergien zu unterscheiden [28]: Die Typen I bis III werden durch Antikörper vermittelt. Dies ist der Fall bei Heuschnupfen, Tierhaarallergien, allergischem Asthma oder bei Lebensmittelallergien.

\section{Hautprobleme bei Musikern \\ $\nabla$}

Bei Musikern tritt die allergische Kontaktdermatitis, auch Kontaktekzem oder Urtikaria, als besonderes Krankheitsbild auf ( Abb.1). Ausgelöst wird das Kontaktekzem durch eine verzögerte Immunreaktion als spezifische Antwort auf ein auf die Haut einwirkendes Kontaktallergen [4]. Zahlreiche Materialien können bei empfindlichen Personen bei andauerndem Hautkontakt eine Kontaktallergie auslösen. Beim Musizieren hat der Musiker mit unbedeckten Hautpartien Kontakt zum Instrument. An erster Stelle sind hier die Blasinstrumente zu nennen. Problematisch ist, dass bei fast allen Holzblasinstrumenten die besonders empfindliche Haut der Lippen und Mundschleimhäute direkten Kontakt zum Instrument hat [9]. Beim Spiel der Querflöte [11] und bei Blechblasinstrumenten sind die Hautpartien rund um die Mundregion betroffen. Auch die hohen Streichinstrumente können diesbezüglich Probleme bereiten. Hier bestehen großflächige Hautkontakte zum Instrument im Bereich der linken, unteren Gesichtspartien, am Kinn und am Hals [13]. Besonders bekannt ist in diesem Zusammenhang der „Geigenfleck“, der häufig als allergische Reaktion auf das Material des Kinnhalters bei Geigern und Bratschisten zu beobachten ist. Typische Symptome einer allergischen Kontaktdermatitis sind Rötung, Schwellung, Knötchen, Quaddeln und Bläschen oder auch Schuppung an den Hautpartien, die direkten Kontakt mit dem Allergen haben. Dabei verläuft die Ekzemreaktion, besonders bei andauerndem und wiederholtem Kontakt, ausgehend von Bläschen und nässenden Erythemen bis hin zu trockener Schuppung oder schmerzhaften Hautrissen in Stadien. Die betroffenen Hautpartien können mitunter heftig jucken. Bei schweren Reaktionen kommt es zu Entzündungsreaktionen mit brennenden Schmerzen, etwa vergleichbar mit einem Sonnenbrand. In schweren Fällen können diese typischen Hautreaktionen auch über die Kontaktstellen hinaus auftreten. Wird der Kontakt zum Allergen nicht abgestellt, kann das Ekzem chronisch werden, worauf sich eine Vergröberung der Haut und übermäßige Verhornung einstellen.

Bei der Kontaktdermatitis handelt es sich speziell um eine allergische Reaktion vom Typ IV, dem Spättyp, auch „Antikörper-unabhängiger Typ“. Bei dieser Art der Allergie wird die Reaktion durch die Aktivierung Allergen-spezifischer T-Zellen ausgelöst. Angesichts der Tatsache, dass Musikinstrumente entweder aus Holz oder Metall-Legierungen bestehen, ist es erwähnenswert, dass die Kontaktdermatitis auf zwei unterschiedlichen Mechanismen beruht:

Bei der Reaktion vom Typ IVa1 richtet sich die Reaktion gegen lösliche Antigene und führt zur Aktivierung von Makrophagen (TH1-Zellen). Diese Reaktion ist zum Beispiel Auslöser der Nickeloder Kobalt-Kontaktdermatitis, von der häufig Querflötenspieler und Blechbläser betroffen sind [50]. Nickel und Kobalt werden zur Optimierung der Material- und Klangeigenschaften den für Querflöten oder Blechblasinstrumente verwendeten Metallen beigefügt oder auch ersatzweise für Edelmetalle wie Silber ver-

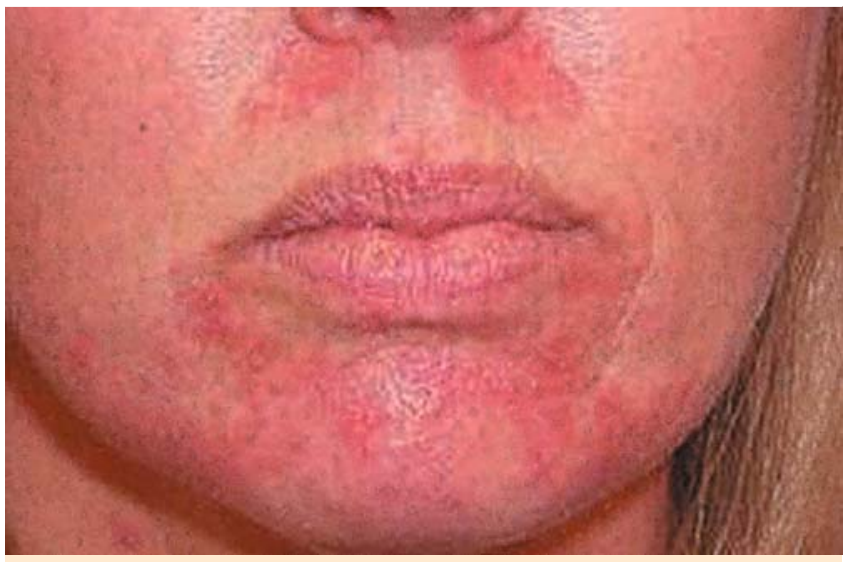

Abb. 1 Hochgradige Kontaktdermatitis bei einer Blechbläserin.

wendet. Vor allem preisgünstige Instrumente für Anfänger enthalten in hohem Maße Nickel.

Die Reaktion vom Typ IVa2 richtet sich gegen lösliche Antigene und führt zur Aktivierung von eosinophilen Granulozyten (TH2Zellen). Dies ist besonders bei Reaktionen auf in Hölzern enthaltenen Allergenen der Fall, die sich nach längerem Hautkontakt durch Erwärmung oder durch Schweiß und Speichel aus dem Material herauslösen [28]. Weil der mechanische Kontakt zum Material sowie die Feuchte von Schweiß und Speichel die Barriere-Funktion der Haut zusätzlich schwächen, können die Allergene nach einiger Zeit vermehrt in tiefere Hautschichten vordringen [2].

Chemisch betrachtet sind Kontaktallergene niedermolekulare Verbindungen, die mit Proteinen der Haut reagieren. Erkannt wird vom Immunsystem ein Bruchstück eines Moleküls, das aus einem Teil des Kontaktallergens und einem Peptid aus dem ursprünglichen Protein besteht. Eine Kontaktallergie läuft immer in zwei Schritten ab: Zunächst kommt es zu einer komplexen Sensibilisierungsphase, die fast immer unbemerkt verläuft. In den Lymphknoten werden die spezifischen T-Zellen aktiviert. Sie vermehren sich und stehen von nun an „auf dem Wachtposten“, um bei erneutem Kontakt mit dem Allergen schnell aktiviert zu werden. Die Zellen zirkulieren mit dem Blutstrom durch den Körper. Sie sammeln sich im Gewebe der Haut, zu der sie aufgrund spezifischer biochemischer Signale eine hohe Affinität besitzen. Der Sensibilisierungsphase folgt die Auslösephase, bei der die beschriebenen Hauterscheinungen auftreten. Kommt es erneut zu einem Kontakt mit dem Allergen, tritt die Auslösephase ein. Dabei schütten die spezifischen T-Zellen immunologische Botenstoffe aus, die die Zellen veranlassen, ein entzündliches Zellinfiltrat aufzunehmen. Diese Botenstoffe und das Zellinfiltrat sind letztlich Auslöser für das sicht- und spürbare Ekzem. Typischerweise tritt die Hautreaktion bei bereits eingetretener Sensibilisierung erst 2-3 Tage nach dem erneuten Allergenkontakt auf, was zu der Bezeichnung „Spättypreaktion“ führte. Darin liegt die Ursache, weshalb viele Betroffene oft lange die lästigen Ekzeme mit sich herumtragen, ohne zu ahnen, welches Material Schuld an ihren Beschwerden haben könnte.

Einmal ausgereift und aktiviert haben die allergenspezifischen T-Zellen sprichwörtlich ein „langes Gedächtnis“ („Persistenz“). Das bedeutet, dass sogar nach jahrelangem Meiden des allergieauslösenden Materials die Allergie bei erneutem Kontakt wieder auftreten kann. Dabei fungiert das Komplementsystem als Über- 
mittler zwischen der Sensibilisierung und der erneuten Auslösung bei wiederholtem Kontakt. Dabei konnte eine besondere Aktivität des C3aR-Rezeptors während der akuten Entzündungsreaktion der Haut nachgewiesen werden [37].

\section{Besonderes Risiko durch Tropenhölzer}

Besonders häufig sind bei Musikern, aber auch in der Holzverarbeitung und im Instrumentenbau [1] die sog. Tropenholzallergien [14]. Generell können alle botanischen Holzspezies allergieauslösend wirken, jedoch ist das Risiko bei den tropischen Arten besonders hoch [12]. Im holzverarbeitenden Gewerbe wie Musikinstrumentenbau, Bootsbau und in der Möbelindustrie kommt als Risikofaktor hinzu, dass die im Holz enthaltenen Allergene durch den Holzstaub in die Luft gelangen [10] und teilweise eingeatmet werden [40]. Inzwischen sind die allergieauslösenden pflanzlichen Stoffe gut bekannt und klassifiziert. Entsprechend der chemischen Struktur des Allergens und des jeweiligen Pflanzenstoffes zeigt sich im Falle einer allergischen Reaktion ein höchst unterschiedliches klinisches Bild. Die allergieauslösenden pflanzlichen Allergene lassen sich entsprechend ihrer chemischen Struktur in 5 Hauptgruppen einteilen. Durch das bessere Verständnis der besonderen Strukturen dieser chemischen Stoffgruppen konnten die Mechanismen der allergischen Kontaktreaktionen insgesamt dargestellt und auch die vielfach auftretenden Kreuzreaktionen auf chemisch verwandte Stoffe besser verstanden werden [42]. Unter allen Instrumentalisten haben Blockund Querflötenspieler [17], Oboisten und Klarinettisten, aber auch Perkussionisten ein besonderes Risiko für eine Kontaktallergie. Hintergrund ist die Tatsache, dass zum Bau hochwertiger Holzblasinstrumente sowie für Klöppel in der Regel tropische Hölzer verwendet werden. Tropenholz unterscheidet sich von den einheimischen Holzarten durch seine extreme Härte sowie durch längere Haltbarkeit und besondere Klangeigenschaften [27]. Vor allem die Ebenholz- und Palisanderarten besitzen einen besonders kräftigen und durchsetzungsfähigen Klang [25]. Als das edelste Holz für Holzblasinstrumente (Flöten, Oboen, Klarinetten) gilt das in Tansania und Mosambique wachsende Grenadil (Dalbergia melanoxylon): Es gehört zu den Palisanderarten, ist schwarz gefärbt und außergewöhnlich hart [52]. Professionelle Spieler bevorzugen daher, etwa wenn sie von einem Orchester begleitet werden, ein Instrument aus Palisander, Ebenholz oder Grenadil [46]. Als besonders aggressiver Allergieauslöser gilt das aus Südamerika stammende Coccobolo (Dalberghia retusa) [33]. Alle diese Rosenholzgewächse der Gattung Dalberghia enthalten sog. Neoflavonoide, darunter den Stoff MethoxyDalbergion [5,21]. Dieser den Bezochinonen zugeordnete Stoff gilt als hochaggressives Allergen. Es wirkt gegenüber Reticulitermes flavipes toxisch, allerdings hat es auf verschiedene holzzerstörende Pilze keinerlei Wirkung [6].

Einige Autoren gehen sogar davon aus, dass regelmäßige, dauerhafte Konfrontation mit diesem Allergen irgendwann bei jeder Person eine Allergie auslösen kann [30]. Generell kann gesagt werden, je länger und je häufiger der Hautkontakt zu einem bestimmten Material besteht, desto größer ist die Wahrscheinlichkeit, dass es zu einer allergischen Reaktion kommt. Das individuelle Risiko einer Person hängt dabei auch vom gesundheitlichen Allgemeinzustand und von Zustand seines Immunsystems sowie von genetischen Faktoren ab. Berufsmusiker haben aufgrund ihrer beruflichen Tätigkeit und längeren Übezeiten weitaus mehr Kontakt mit dem Instrument als Laien- oder Hobby-Musi- ker, weshalb sie weitaus häufiger betroffen sind und dementsprechend auch ein viel größeres Risiko haben, im Laufe ihres Musikerlebens eine Kontaktallergie zu entwickeln [7]. Wie hoch die Prozentzahl von Musikern (Laien und Berufsmusiker) ist, die unter einem Kontaktekzem gegen Tropenhölzer, Nickel oder Kolophonium leiden, ist nicht bekannt. Es wurden zahlreiche Untersuchungen über mögliche dermatologische Erkrankungen bei Musikern durchgeführt [50], allerdings können aus den Ergebnissen keine allgemeinen Prozentzahlen abgeleitet werden. In der Literatur existieren etliche Fallbeschreibungen von betroffenen Instrumentalisten, darunter Klarinettisten, Saxophonisten, Block- und Querflötenspieler, Blechbläsern und Streichern, jedoch ohne zuverlässige Häufigkeitsangaben [4]. Würde man systematisch verschiedene Musiker eines Orchesters auf mögliche Hautveränderungen untersuchen, so ließen sich sicher zahlreiche positive Befunde feststellen.

Viele Betroffene suchen erst dann einen Facharzt auf, wenn das Kontaktekzem bereits ein höhergradiges Stadium erreicht hat. So kann der sog. „Geigenfleck“ an der linken Kinn-/Halsseite von Violonisten und Bratschisten häufig beobachtet werden [19]. Geigen und Bratschen sind heute im Allgemeinen an ihrer unteren, linken Korpusseite mit einem Kinnhalter versehen, der das Halten des Instruments erleichtert. Dabei ruhen Kinn- und Unterkieferpartie des Spielers in dem entsprechend geformten Kinnhalter, der meistens passend zum Instrument aus Ebenholz oder Palisander besteht. Kommt es zu einer Reaktion auf das Material, entwickelt der Spieler an seiner linken Hals- und Kinnpartie den typischen „Geiger-Fleck“ [12]. Offensichtlich haben zahlreiche Musiker ein Kontaktekzem, ohne dass eine Diagnose gestellt wird und eine Behandlung erfolgt. Solange die kosmetischen, gesundheitlichen und spieltechnischen Einschränkungen nicht zu gravierend sind, werden derartige Begleitumstände des Musikerberufes unkritisch in Kauf genommen. Ebenso wenig kann eindeutig gesagt werden, welches Musikinstrument im Hinblick auf eine Kontaktallergie das größte Risiko darstellt. Ein klares Kriterium ist diesbezüglich die Art und die Menge der verwendeten Materialien: Instrumente, deren Spiel großflächigen Kontakt zu möglichen allergenen Stoffen erfordert, stellen sicher ein höheres Risiko dar. Betrachtet man die Ergebnisse aus der Literatur, so sind Holzblasinstrumente, Trommelschlägel sowie Kinnhalter von Violinen und Bratschen die häufigsten Gründe für eine Kontaktdermatitis beim Musiker. Das geringste Risiko, eine Kontaktallergie zu entwickeln, haben sicher Pianisten, da sie nur mit ihren Fingerkuppen Kontakt zum Instrument haben und die Klaviertasten mit unbedenklichen Materialien wie Elfenbein oder Elfenbeinimitat (Kunststoff) belegt sind.

\section{Mögliche Maßnahmen zum Schutz vor Hautreaktionen $\nabla$}

Der Musiker sollte sich über Risiken und Inhaltsstoffe bestimmter Holzarten genau informieren und ggf. den Kontakt zu dem Material vermeiden. Die meisten Holzblasinstrumente - auch Oboen und Klarinetten - werden heute auch aus europäischen Holzarten gebaut, die weitgehend unbedenklich sind. Im Fall einer Allergie kann der Spieler auf diese Holzarten zurückgreifen. Als besonders hautfreundlich gilt der europäische Buchsbaum (Buxus semper virens), aber auch Obsthölzer sind unbedenklich. Der Erwerb eines Zweitinstruments aus diesen unbedenklichen Materialien ist für Berufsmusiker auf jeden Fall sinnvoll.

Bei den Holzblasinstrumenten bietet sich an, das Mundstück aus einem neutralen Material anfertigen zu lassen. Bei Blockflöten 


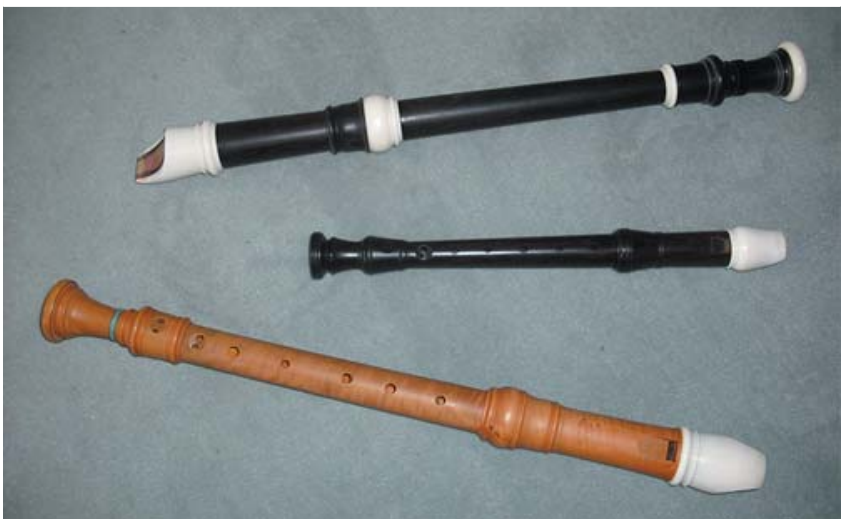

Abb.2 Blockflöten aus Ebenholz (oben, Mitte) und europäischem Buchsbaum (unten) mit Elfenbein-Ummantelung des Mundstückes (Nachbauten barocker Originale aus der Sammlung der Autorin, Foto der Autorin).

kann der Schnabel mit einer Ummantelung aus Mammutelfenbein $(\bullet$ Abb.2) oder einem Spezialkunststoff verkleidet werden. Auch hautverträgliche Lacke bieten sich dort an, wo die Hände und andere Hautpartien Kontakt zum Instrument haben; etwa bei Flöten, Trommelschlägeln oder Kinnhaltern für Geigen und Bratschen. Diese Zubehörteile sind auch aus europäischen Holzarten und Kunststoffen im Handel erhältlich. Gegebenenfalls bietet sich auch die Möglichkeit, das Instrument an den Kontaktstellen mit einem hautverträglichen Lack zu lackieren und so mit einem Schutzanstrich zu versehen. Das Problem bei dieser Maßnahme ist allerdings, dass Dalbergione die Trocknung von Ölund Polyesterlacken behindern [43] und dass die Allergene durch die Lackschicht diffundieren und somit ebenfalls an die Haut gelangen [5].

\section{Gefährdung durch mögliche Kreuzreaktionen}

Für Musikpädagogen ist es wichtig, die Eigenschaften der verschiedenen Hölzer zu kennen, damit sie ihre Schüler beraten und vor einer Allergie schützen können. Besonders Blockflötenlehrer, die es häufig mit noch sehr jungen Schülern zu tun haben, sollten sich informieren und die Eltern beim Kauf eines Instruments beraten. Für Anfänger werden in der Regel zunächst preisgünstige Flöten aus Ahorn oder Birnbaum gekauft. Wird für fortgeschrittene Schüler der Kauf eines hochwertigen Instruments erwogen, sollte der Lehrer Eltern und Schüler diesbezüglich aufklären. Sinnvoll ist die Frage, ob der Schüler schon eine Allergie, etwa auf Blütenpollen oder Lebensmittel, hat: Ist dies der Fall, sollte man tropische Holzarten meiden, denn wenn schon eine Allergie besteht, ist die Wahrscheinlichkeit groß, auch gegen neue, hochaggressive Allergene eine Sensibilität zu entwickeln [29]. In diesem Zusammenhang spielt das Phänomen der Kreuzreaktionen eine entscheidende Rolle: Eine Kreuzreaktion kommt dann zustande, wenn Immunglobulin-E-Antikörper, die gegen ein bestimmtes Allergen gerichtet sind, auch andere, chemisch verwandte Allergene [44] in anderen Allergenquellen erkennen und bei Kontakt mit diesen ebenfalls eine allergische Reaktion auslösen [51]. Beispielsweise kann es bei einer bestehenden Allergie gegen Primelpollen bei Kontakt mit einigen exotischen Holzarten, die Chinone mit verwandten Strukturen enthalten, zu Kreuzallergien kommen [45]. Eine solche Kreuzreaktion könnte auftreten, wenn bei einer vorhandenen Allergie gegen Primelpollen und andere Gewächse aus der Gruppe der Heidekraut- artigen ein Blasinstrument oder Kinnhalter aus Palisander verwendet wird. In diesem Fall löst das R-Enantiomer, nicht aber das S-Enantiomer die Allergie aus [16]. Wieviele Instrumentalisten mit einer bestehenden Allergie gegen Blütenpollen oder Nahrungsmittel eine Kreuzreaktion auf die im Instrumentenbau verwendeten Materialien entwickeln, ist nicht bekannt. Diesbezügliche Zahlen liegen nicht vor.

\section{Gefährdung durch Alltagsgegenstände \\ $\nabla$}

Für betroffene Musiker wie auch für den Arzt ist es daher unbedingt erforderlich zu wissen, in welchen Materialen oder Gegenständen die oben erwähnten Kontaktallergene möglicherweise vorkommen. Aufgrund ihrer dekorativen Maserung werden tropische Holzarten heutzutage gerne für alle möglichen Gebrauchsgegenstände verarbeitet, wie etwa Modeschmuck, Salatschüsseln oder Frühstücksbrettchen. Daher kann das Tragen von Schmuckstücken aus tropischem Holz nach längerem Kontakt ebenso eine Kontaktdermatits auslösen [15]. Musikerinnen sollten auf jeden Fall auf das Tragen derartiger Schmuckstücke verzichten, denn wenn sie gegen das Material des Schmuckes eine Allergie entwickeln, werden sie auch auf den Kontakt mit ihrem Instrument allergisch reagieren, sofern es aus demselben oder einem botanisch verwandten Material gefertigt ist! Auch von der Verwendung von Haushaltsgegenständen, die ggf. mit Lebensmitteln in Berührung kommen, wie z.B. Frühstücksbrettchen oder Salatschüsseln, ist in dieser Hinsicht abzuraten. Ein anderer Schwerpunkt bei der Verarbeitung von Tropenhölzern ist die Möbelindustrie. Meistens werden hier aus Kostengründen nur Furniere verwendet. Im Hinblick auf Kontaktallergien können Möbel sicher weitgehend als unbedenklich bezeichnet werden, es sei denn, man kommt beispielsweise an Schreibtischen dauerhaft und wiederholt mit Partien der Hände und Unterarme mit der Holzoberfläche in Kontakt. In den meisten Fällen sind jedoch Möbel lackiert oder auf andere Art versiegelt, sodass eine Kontaktallergie in diesem Zusammenhang eher unwahrscheinlich ist.

\section{Allergische Reaktionen durch Lacke und Harze $\nabla$}

Bei Streichinstrumenten können die im Geigenlack enthaltenen Inhaltsstoffe Allergien auslösen. Geigenlack besteht aus verschiedenen Harzen wie Sandarak, Kopale, Safran, Weihrauch, Kolophonium und Propolis. Als Lösungsmittel wird Alkohol verwendet [24]. Besonders bei neuen Instrumenten, deren Lack noch ausdünstet, kann dies der Fall sein. Professionelle Spieler spielen in der Regel alte Instrumente, deren Lack keine Ausdünstungen mehr abgeben wird. Benutzt der Spieler ein Schulterkissen oder -stütze sind die Hautpartien, mit denen er Kontakt zu den lackierten Flächen der Geige hat, ohnehin begrenzt. Durch die Wahl eines hautverträglichen Materials kann dem vorgebeugt werden. Das im Geigenlack enthaltene Propolis, ein von Bienen produziertes Wachs-Harz, war schon im Alten Ägypten als Heilmittel und Werkstoff bekannt [26]. Auch Hildegard von Bingen erwähnt in ihrer Heilkunde „die Kraft der Bienen“ [22]. Aus diesem Grund wird es auch heute noch häufig als Zusatz in naturheilkundlichen und pflanzlichen Heilmitteln, aber auch in Pflegeprodukten für Haut und Haare, Cremes und Salben verwendet [49]. Äußerlich wird es bei Verletzungen der Haut, Sonnenbrand, Ekzemen oder in der medizinischen Fußpflege eingesetzt. In 
Lutschpastillen wird es zum Schutz vor Infektionen und zur Behandlung von Erkältungskrankheiten und Entzündungen der unteren Atemwege zugesetzt. Traditionell gilt es als Stärkungsmittel der Immunabwehr [41]. Patienten mit einem allergischen Kontaktekzem auf Propolis begegnen dieser Substanz daher noch in zahlreichen anderen Heil- und Gebrauchsmitteln. Allergische Reaktionen auf diesen Stoff sind in der jüngeren Zeit häufig dokumentiert worden [18]. In einer klinischen Studie an 7483 gesunden Probanden zeigten zwischen 1,2 bis 3,3\% der Personen bei Hautkontakt eine allergische Reaktion [20]. Auch in anderen Berufsgruppen kommen allergische Reaktionen auf Propolis vor: In einer Studie an 1051 Imkern wurden 37 Betroffene (3,6\%) ermittelt [35]. Dabei zeigte sich, dass die Mehrzahl der Betroffenen auch auf andere Allergene vermehrt ansprachen [36].

Ebenso kritisch ist das Kolophonium zu betrachten, das auf die Bogenhaare ( $\bullet$ Abb.3) aufgetragen wird. Beim Spielen wird es von den Haaren abgerieben und verteilt sich als feiner Staub in die Umgebung. Weil Kolophonium heftige Reaktionen auslösen kann, sowohl auf der Haut als auch durch Einatmung, sollte man nicht zu großzügig mit diesem Stoff umgehen. Neben der Verwendung als Bogenharz kommt es als Zusatzstoff in zahlreichen Klebstoffen, Lacken sowie Neopren für Taucheranzüge vor und dient als Flussmittel für Elektronik-Lötarbeiten. Auch als Räucherharz in der Lebensmittel- und als Weichmacher in der Textilindustrie oder zur Oberflächenoptimierung von Sportgeräten wie Handbällen oder Holmen wird es verwendet. Außer bei Musikern sind auch auf diesen Stoff allergische Reaktionen bei unterschiedlichen Gruppen dokumentiert [38, 39].

\section{Diagnose durch Epikutantest}

$\nabla$

Grundsätzlich sollte der Musiker bzw. Eltern und Lehrer darauf achten, ob sich an den am Instrumentalspiel beteiligten Hautflächen Auffälligkeiten wie Rötung oder Bläschen zeigen. In diesem Fall sollte beim Hautarzt ein Epikutantest durchgeführt werden. Dabei werden die verdächtigen Kontaktallergene mit speziellen Testpflastern für $48 \mathrm{Std}$. auf die Rückenhaut geklebt. Bei den Stoffen, auf die der Patient allergisch reagiert, entwickelt sich an der Teststelle die typische Ekzemreaktion [53]. Für die Testung von Metalllegierungen und Chemikalien stehen dem Arzt fertige Testmuster zur Verfügung. Bei Verdacht auf Tropenholzallergien muss der Patient das Testmaterial ggf. selbst besorgen. Dazu bittet man Instrumentenbauer um Proben mit Holzstaub, der beim Drechseln, Bohren oder Schleifen im Rahmen des Herstellungsprozesses anfällt. Der Instrumentenbauer sollte die Staubproben der verschiedenen Holzarten in geeignete Behälter wie kleine Tütchen oder Reagenzgläser abfüllen. Wenige Gramm reichen für den Test aus. Wichtig ist, dass die Proben relativ rein sind: d.h. wo Palisander drauf steht, sollte auch nur Palisander drin sein. Ansonsten können die Testergebnisse verfälscht werden [8].

Zeigt der Test positive Reaktionen auf bestimmte Holzarten, muss der Musiker auf jeden Fall den Kontakt zu dem Material vermeiden und auf andere Materialien zurückgreifen oder das vorhandene Instrument mit entsprechendem Schutz versehen. Bei großflächigen Entzündungsreaktionen kann eine kurzzeitige Therapie mit einem Glukokortikosteroid erfolgen. In der Regel wird dieses als Salbe auf die betroffenen Hautstellen aufgetragen. In besonders schweren Fällen kann eine systemische Therapie in Form von Tabletten oder Injektionen notwendig sein [47]. Dem praktischen Arzt oder betroffenen Musiker soll die $\bullet$ Tab. 1 im

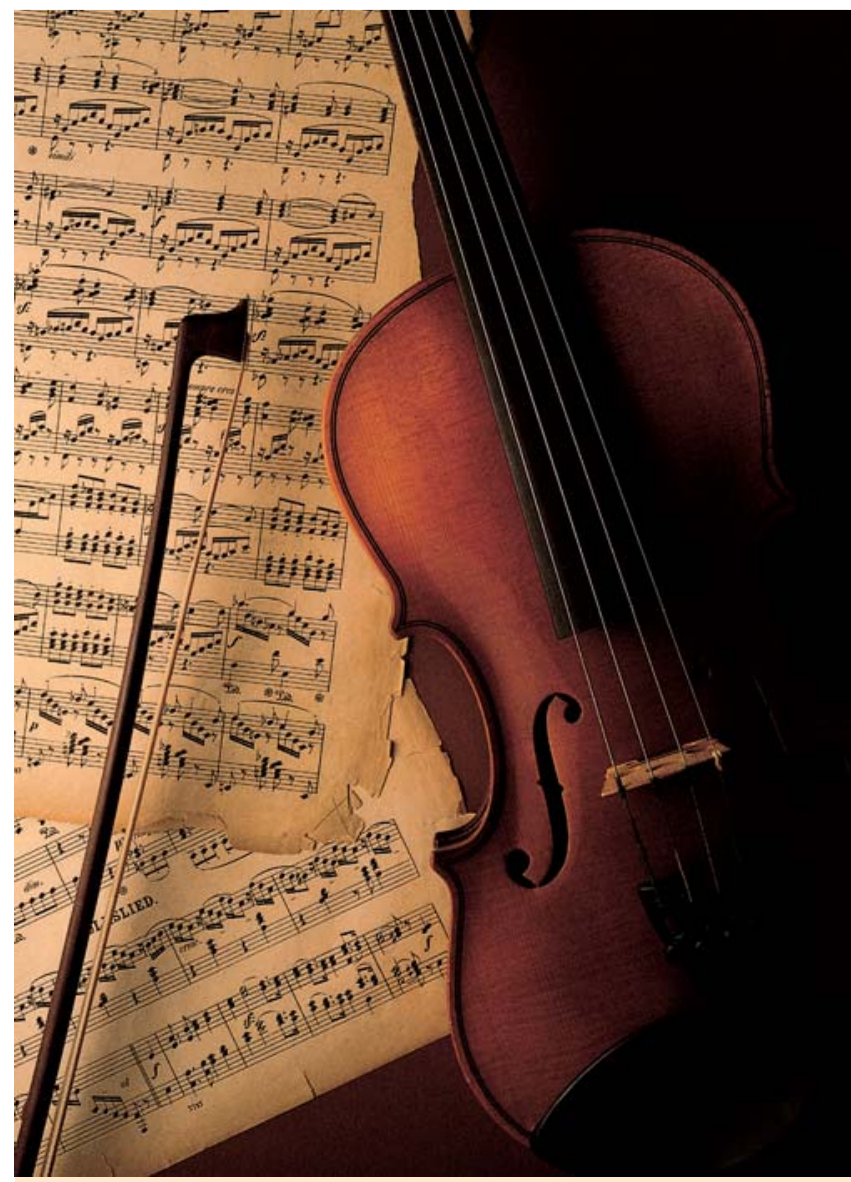

Abb.3 Bei Streichinstrumenten können das auf die Bogenhaare aufgetragene Kolophonium und die im Geigenlack enthaltenen Harze Allergien und eine Kontaktdermatitis auslösen (Quelle: ccvision).

Falle eines Allergieverdachts als Orientierungshilfe und zur Information dienen.

\section{Probleme durch fehlende medizinische Aufklärung und Betreuung $\nabla$}

Die Frage nach dem Fazit für die ärztliche Praxis gestaltet sich vielseitig, da viele Musiker den Arzt erst aufsuchen, wenn sie bereits deutliche Symptome zeigen und selber einen Verdacht haben. Der praktische Arzt sollte Patienten, die deutliche Hautsymptome an Gesicht, Hals oder an den Händen zeigen, auf mögliche Ursachen oder besondere Aktivitäten ansprechen. Hausärzte kennen die Berufe ihrer Patienten und würden daher einen Musiker mit auffälligen Ekzemen zum Facharzt überweisen. Grundsätzlich sollte der Musiker seinen Arzt über seinen Beruf und die gesundheitlichen Risiken des Berufsmusikers informieren, denn nur dann kann der Arzt bei evtl. auftretenden Problemen sinnvolle Maßnahmen ergreifen und den Patienten an einen Spezialisten überweisen. Anders sieht es bei Laienmusikern aus, die oft ebenfalls viele Stunden mit ihrem Instrument verbringen. Bei ihnen weiß der Hausarzt oft nicht von ihrem musikalischen Hobby und eine geeignete Beratung wird daher versäumt. In diesem Fall lohnt es sich nachzufragen, damit der Patient eine Weiterbehandlung erfährt.

Daher soll an dieser Stelle auch ein Fazit für die musikpädagogische Praxis nicht fehlen: Hier liegt es vor allem in der Verantwor- 
Tab. 1 Übersicht über die verschiedenen Eigenschaften und pathogenen Inhaltsstoffe der im Musikinstrumentenbau verwendeten Holzarten [27, 31].

\begin{tabular}{|c|c|c|c|c|}
\hline Holzart & Herkunft & Eigenschaften $[27,31,32]$ & Pathogene Inhaltsstoffe [5] & Allergie-Risiko [30] \\
\hline $\begin{array}{l}\text { Ahorn (Acer } \\
\text { pseudoplatanus) }\end{array}$ & Europa, Nordamerika & $\begin{array}{l}\text { Fest feinporig, warmer, grund- } \\
\text { töniger Klang }\end{array}$ & $\begin{array}{l}\text { Tannine, Phytosterin, Allantoin, } \\
\text { Flavonoide, Saponine, Cholin }\end{array}$ & 0 \\
\hline Birne (Pirus communis) & Europa & $\begin{array}{l}\text { Fest, fein, gleichmäßig, warmer, } \\
\text { reiner, grundtöniger Klang }\end{array}$ & Gerbstoffe, Proanthocyanidine & o \\
\hline $\begin{array}{l}\text { Pflaume (Prunus } \\
\text { domestica) }\end{array}$ & Europa & $\begin{array}{l}\text { Hart, dicht, optisch edel, warmer, } \\
\text { tragender Ton }\end{array}$ & Prunasin, Sorbitol & o \\
\hline Kirsche (Prunus avium) & Europa & Fest, fein, warmer, weicher Ton & Prunasin, Sorbitol & 0 \\
\hline Olive (Olea europaea) & Südeuropa & $\begin{array}{l}\text { Hart, dicht, optisch edel, warmer, } \\
\text { satter Ton }\end{array}$ & Glycerin, Palmitoleinsäure & o \\
\hline $\begin{array}{l}\text { Europäischer Buchs- } \\
\text { baum (Buxus semper } \\
\text { virens) }\end{array}$ & Mittel-u. Südeuropa & $\begin{array}{l}\text { Sehr hart, gleichmäßig dicht, } \\
\text { warmer, kräftiger, tragender Ton }\end{array}$ & Cyclobuxin D & 0 \\
\hline $\begin{array}{l}\text { Indisch Buchs } \\
\text { (Gardenia latifolia) }\end{array}$ & Indien & Hart, dicht, klarer, voller Ton & Chinone (Latinon) & + \\
\hline $\begin{array}{l}\text { Rosenholz (Dalberghia } \\
\text { variabilis) }\end{array}$ & Südamerika & $\begin{array}{l}\text { Sehr hart u. dicht, optisch } \\
\text { dekorativ, warmer, käftiger, } \\
\text { tragender Klang }\end{array}$ & (R)-4-Methoxydalbergion & ++ \\
\hline $\begin{array}{l}\text { Rio Palisander } \\
\text { (Dalbergia nigra) } \\
\text { Ostindischer Palisander } \\
\text { (Dalbergia latifolia) }\end{array}$ & Südamerika, Indien & $\begin{array}{l}\text { Sehr hart u. dicht, obertonreich, } \\
\text { strahlender, klarer Ton }\end{array}$ & $\begin{array}{l}\text { (R)-+(S)-Methoxydalbergion, } \\
\text { andere Dalbergione }\end{array}$ & +++ \\
\hline $\begin{array}{l}\text { Iroko/Kambala } \\
\text { "African Teak" (Chloro- } \\
\text { phora excelsa) }\end{array}$ & Zentral- u. Ostafrika & $\begin{array}{l}\text { Sehr hart u. dicht, warm u. kräftig, } \\
\text { in großen Stücken lieferbar, oft } \\
\text { für tiefe Instrumente verwendet. }\end{array}$ & Chlorophorin & ++ \\
\hline $\begin{array}{l}\text { Cocobolo (Dalberghia } \\
\text { retusa) }\end{array}$ & Südamerika & $\begin{array}{l}\text { Sehr hart, rötliche Färbe, edel } \\
\text { gemasert, kräftiger, klarer, } \\
\text { tragender Ton, durchsetzungs- } \\
\text { fähige, weiche Dynamik }\end{array}$ & $\begin{array}{l}\text { Obtusachinon, (R)-4-Methoxy- } \\
\text { dalbergion, andere Dalbergione }\end{array}$ & $\begin{array}{l}+++(!) \text { Höchstes Risiko! } \\
\text { Gilt als „giftig“! }\end{array}$ \\
\hline $\begin{array}{l}\text { Grenadill (Dalberghia } \\
\text { melanoxylon) }\end{array}$ & Zentral-u. Ostafrika & $\begin{array}{l}\text { Härtestes im Instrumentenbau } \\
\text { verwendetes Holz, sehr dicht, } \\
\text { gleichmäßig, kräftiger, edler } \\
\text { Klang, edle schwarze Farbe }\end{array}$ & $\begin{array}{l}\text { (S)-4-Methoxydalbergion, } \\
\text { (S)-4'-Hydroxy-4-methoxy- } \\
\text { dalbergion }\end{array}$ & +++ \\
\hline $\begin{array}{l}\text { Ebenholz (Diospyros } \\
\text { melanoxylon) }\end{array}$ & $\begin{array}{l}\text { Indien, Tropisch } \\
\text { Westafrika }\end{array}$ & $\begin{array}{l}\text { Sehr hart u. dicht, gleichmäßig, } \\
\text { tiefschwarze Farbe, kräftiger, } \\
\text { edler, strahlender Klang }\end{array}$ & $\begin{array}{l}\text { Makassarchinon, } \\
\text { (R)-+ (S)-Methoxydalbergion }\end{array}$ & +++ \\
\hline
\end{tabular}

Allergie-Risiko: +++ hoch, ++ mittel, + gering, o neutral/unbedenklich

Die angegebenen Risiko-Werte spiegeln Erfahrungswerte wider, die zum einen auf Angaben der botanischen Fachliteratur [8] als auch auf Erfahrungen und Aussagen von betroffenen Musikern und Instrumentenbauern basieren. Das individuelle Risiko hängt von der jeweiligen Person, dem Zustand ihres Immunsystems und verschiedenen anderen Faktoren ab.

tung des Instrumentalpädagogen, Eltern, Schüler und Studenten über den umsichtigen Umgang mit tropischen Hölzern aufzuklären. Gerade bei Allergien gilt das alte Sprichwort „Vorbeugung ist besser als Heilen“. Hier sind besonders Kinderärzte dazu aufgerufen, Eltern entsprechend zu beraten. Ziehen Eltern das Erlernen eines Instruments für ihr Kind in Betracht, berichten sie oft dem Kinderarzt von ihrem Vorhaben. Einige Ärzte raten sogar zum Instrumentalunterricht, um die geistige und feinmotorische Entwicklung eines jungen Patienten zu fördern. Bei der Wahl des Instruments sollte auf gesundheitlich unbedenkliche Materialien geachtet werden. In den letzten Jahren wurde der Markt mit einer Flut von preisgünstigen Instrumenten aus China und anderen Billiglohnregionen Asiens überschwemmt. In den letzten Jahren steigen chinesische Konzerne zunehmend in den Musikinstrumentenmarkt ein. Sogar die Produktionsraten von Blockflöten steigen dort rasant. Im Vergleich zu europäischen Produkten werden diese Instrumente zu erschreckend niedrigen Preisen angeboten [48]. Gerade für Kinder kaufen Eltern häufig ein Instrument der untersten Preiskategorie. Problematisch an diesen Billigimporten sind nicht nur ihre mangelnde klangliche und handwerkliche Qualität, sondern auch die verwendeten Materialien, Klebstoffe und Lacke, deren Inhaltsstoffe nicht bekannt sind und die unseren gesundheitlichen Standarts oftmals nicht entsprechen. Besonders Streich- und Holzblasinstrumente aus China bestehen zum Teil aus uns unbekannten Hölzern oder enthalten giftige Lacke [48]. Während der Profimusiker durch die Verwendung tropischer Materialien und der berufsbedingt langen täglichen Spieldauer gefährdet ist, eine Kontaktallergie zu entwickeln, stellt bei Anfängern und Kindern eher die Unwissenheit von Eltern und Lehrern sowie die Wahl von Instrumenten unbekannter Hersteller und Materialien ein Risiko dar. Hier sollte der Arzt zu einem Instrument eines hiesigen Herstellers raten, die auch preisgünstige Instrumente anbieten, die besser klingen, besser verarbeitet sind und drittens aus gesundheitlich unbedenklichen Materialien bestehen.

\section{Fazit}

$\nabla$

Kontaktallergien bei Musikern müssen nicht sein. Im Grunde genommen sind sie eine Folge von Unwissenheit und allzu großzügigen Umgangs mit hochallergenen Materialien. Information und verantwortungsvoller Umgang mit tropischen Holzarten sind hier für Musiker, Lehrer, Eltern sowie für den Arzt die beste The- 
rapie. Der Musiker, der seine Karriere und seine Übungseinheiten sorgfältig plant und verantwortungsvoll mit risikobehafteten Materialien umgeht (Schutzmaßnahmen, Zweitinstrumente aus verträglichem Material), kann ein Musiker-Leben lang beschwerdefrei musizieren [3]. Ärzte sollten ihre Musiker-Patienten entsprechend beraten. Auch Instrumentallehrer und Eltern musizierender Kinder sollten diesbezüglich aufgeklärt werden.

\section{Interessenkonflikt}

$\nabla$

Die Autoren geben an, dass kein Interessenkonflikt besteht.

\section{Abstract}

\section{Contact Allergies in Musicians}

During the last years, the problem of allergic diseases has increased. Allergies are errant immune responses to a normally harmless substance. In musicians the allergic contact dermatitis to exotic woods is a special problem. Exotic rosewood contains new flavonoids, which trigger an allergic reaction after permanent contact with the instrument. High quality woodwind instruments such as baroque flute or clarinets are made in ebony or palisander because of its great sound. Today instruments for non-professional players are also made in these exotic materials and non-professionals may have the risk to develop contact dermatitis, too. Brass-player has the risk of an allergic reaction to the different metals contained in the metal sheets of modern flutes and brass instruments. Specially nickel and brass alloys are used to product flute tubes or brass instruments. Special problem arises in children: patients who are allergic to plants or foods have a high risk to develop contact dermatitis. Parents don't know the materials of low-priced instruments for beginners. Often unknown cheap woods from exotic areas are used. Lowpriced brass instruments contain high amount of brass and other cheap metals. Physicians should advice musician-patients or parents about the risks of the different materials and look for the reason of eczema on mouth, face, or hands.

\section{Literatur}

1 Athavale PN, Shum KW, Gasson P et al. Occupational hand dermatitis in a wood turner due rosewood (Dalberghia latifolia). Plant Med 1998; 64: $153-158$

2 de Benedetto A, Kubo A, Beck LA. Skin Barrier Disruption: A Requirement for Allergen Sensitation? J Invest Dermatol 2012; 132: 949-963

3 Blum J. Medizinische Probleme bei Musikern. 1. Aufl.; Stuttgart: Thieme; 1995

4 Bork G. Dermatologische Erkrankungen und Allergien. In: Blum J. Medizinische Probleme bei Musikern. 1. Aufl.; Stuttgart: Thieme; 1995: 228

5 Dietrichs HH, Hausen BM. Dalbergione - Wirkstoffe der Dalbergia-Arten. Holzforschung. Berlin: de Gruyter International Journal of the Biology, Chemistry, Physics and Technology of Wood; 1971: 25 Seiten

6 Chan SC, Chang YS, Wang JP et al. Three new flavonoids and allergic, anti-inflammatory constituents from the heartwood of Dalbergia odorifera. Planta Med 1998; 64: 153-158

7 Claessens N, Le Hermanns T, Piérard-Franchimont C et al. Occupational stigmata in instrumental musicians. Rev Med 2001; 56: $453-455$

8 Forth $W$, Henschler D, Rummel W. Allgemeine und spezielle Pharmakologie und Toxikologie. 9. Aufl.; München: Urban \& Fischer; 2005

9 Fuks L, Byo JL. Wind Instruments. In: Parncutt R, Hrsg. The Science and Psychology of Music Performance. 1. Aufl.; New York: Oxford University Press; 2002: 319-325
10 Gallo R, Guarrera M, Hausen BM. Airborne contact dermatits from east Indian rosewood (Dalberghia latifolia Roxb). Contact Dermatitis 1996; 35: $60-61$

11 Galway J. Die Flöte. 2. Aufl.; Frankfurt: Ullstein Verlag; 1994

12 Gambichler T, Boms S, Freitag M. Contact dermatitis and other skin conditions in instrumental musicians. BMC Dermatol 2004; 4: 3

13 Guettler K, Hallmann S. String Instruments. In: Parncutt R, Hrsg. The Science and Psychology of Music Performance. 1. Aufl.; New York: Oxford University Press; 2002: 303

14 Hallei $N$, Meirion Hughes T, Stone $N$. Contact allergy to thiuram in an musician. Contact Dermatitis 2004; 51: 154

15 Hausen BM. Allergic contact dermatitis from an wooden necklace. Am J Contact Dermat 1997; 8: 185-187

16 Hausen BM, Vieluf K. Allergiepflanzen, Pflanzenallergene. 2. Aufl.; Heidelberg: Ecomed; 1997

17 Hausen BM et al. Cocobolo-Holzallergie bei einer Flötenlehrerin. Akt Dermatol 2004; 30: 489-492

18 Hausen BM. Evaluation of the main contact allergens in propolis (1995 to 2005). Dermatitis 2005; 16: $127-129$

19 Haustein UF. Geigenkinnhalter-Ekzem durch Ostindisch-Palisander (Dalbergia latifolia Roxb.)-Holz. Dermatol Monatsschr 1981; 167: 250

20 Hegyi E, Suchý V, Nagy M. Propolis allergy. Hautarzt 1990; 41: 675679

21 Hegnauer R, Hegnauer M. Chemotaxonomie der Pflanzen. Teil 3: Leguminosae, Papilionoideae. 1. Aufl.; Basel: Birkhauser Verlag; 1963: $125 \mathrm{ff}$

22 von Bingen H. Heilkunde. Von den Heilmitteln. Schippergers H, Hrsg. 6 Aufl.; Salzburg: Otto Müller Verlag; 1992: 263-269

23 Klinke R, Silbernagel S. Lehrbuch der Physiologie. 2. Aufl.; Stuttgart: Thieme; 2000: $201 \mathrm{ff}$

24 Kolneder W. Das Buch der Violine. „Der italienische Lack“. 2. Aufl.; Zürich: Atlantis Musikbuch Verlag; 1989: 154-156

25 Küng A. Die Blockflöte. Schaffhausen: Küng Blockflötenbau; 1990: Copyright: $\mathrm{CH} 8200$

26 Leca AP. Die Medizin im Alten Ägypten. In: Illustrierte Geschichte der Medizin. Band 1. Stuttgart: Bechtermünz Verlag/Lizenzausgabe für Weltbildverlag; 2000: 109-123

27 Linde HM. Handbuch des Blockflötenspiels. 2. Aufl.; Mainz: Schott; 1984: 14

28 Löffler G, Petrides PE, Heinrich PC. Biochemie \& Pathobiochemie. 7. Aufl.; Heidelberg: Springer; 2003: $1155 \mathrm{ff}$

29 Mc Fadden JP, Ingram MJ, Rycroft RJG. Contact allergy to cane reed in a clarinetist. Contact Dermatitis 1992; 27: 17

30 Merfort I. Heil- und Nutzpflanzen mit Haut-Tücken. Pharmazeutische Zeitung 2002; http://www.pharmazeutische-zeitung.de/index.php? id=titel_03_2002 (letzter Zugriff: 9.11.2012)

31 Moeck Musikinstrumente u. Verlag e.K. Gesamtkatalog: Blockflöten für das professionelle Spiel. Klang der Hölzer, Tabelle. Celle; 2010: 3

32 Mollenhauer Blockflötenbau/Conrad Mollenhauer GmbH, 36043 Fulda. Gesamtkatalog „Lust auf Blockflöte“ Wissenswertes: Klangreichtum aus edlen Hölzern. Fulda; 2011: 5

33 Moratinos MM, Tevar E, Conde-Salazar L. Contact allergy to a cocobolo bracelet. Dermatits 2005; 16: 139-141

34 Müller-Esterl W. Molekulare Grundlagen des Immunsystems. In: Müller-Esterl W, Hrsg. Biochemie. 1. Aufl.; Heidelberg: Spektrum Akademischer Verlag; 2004: 458

35 Münstedt K, Hellner M, Hackethal A et al. Contact allergy to propolis in beekeepers. Allergol Immunopathol 2007; 35: 95-100

36 Münstedt K, Kalder M. Contact allergy to propolis in beekeepers. Allergol Immunopathol 2009; 37: 298-301

37 Niebuhr M, Bäumler W, Ketzmann M et al. Participation of Complement 3a receptor (C3aR) in the sensitization phase of Th2 mediated allergic contact dermatitis. Xp Dermatol 2012; 21: $52-56$

38 Ozkaya E, Elinç-Aslan MS, Mirzoyeva L. Allergic contact dermatitis caused by p-tert- butylphenol formaldehyde resin and colophonium in neoprene thermal sauna shorts. Contact Dermatitis 2010; 63: $230-232$

39 Quain RD, Militello G, Crawford GH. Allergic contact dermatitis caused by colophony in an epilating product. Dermatitis 2007; 18: 96-98

40 Rojas-Hijazo B, Lezaun A, Hausen BM et al. Airborne contact dermatitis in gaitas (flageolets) constructors after exposure to sawdust of caviuna. Contact Dermatits 2007; 56: 274-277

41 Rohwedder D, Havsteen BH. Propolis, der Stoff aus dem Gesundheit ist; ein Wirkstoff der Natur. 1. Aufl.; Berlin: BTV-Taschenbuch-Verlag; 1987 
42 Rozas-Munoz E, Lepoittevin JP, Pujol RM et al. Allergic Contact Dermatitis to Plants: Understanding the Chemistry will Help our Diagnostic Approach. Actas Dermosifiliogr 2012; 103: 456-477

43 Sandermann W, Schwarz E. Über den Einfluß von Holzinhaltsstoffen auf die Witterungsbeständigkeit und die Trocknung von Öl- und Polyesterlacken. Farbe u. Lack 1956; 62: 134 - 145

44 Schaeffer M, Talaga P, Stampf JL et al. Cross-reaction in allergic contact dermatitis from alpha-methylene-gamma-butyrolactones: importance of the cis or trans ring junction. Contact Dermatitis 1990; 22: $32-36$

45 Schmalle HW et al. Kontaktallergene, Isolierung, Identifizierung und Struktur- Wirkungsbeziehungen am Beispiel ausgewählter Phytoekzematogene. Funkt Biol Med 1983; 2: 9-17

46 Solum J. The Early Flute. Chap. Buying an Instrument. 1. Aufl.; Oxford: Clarendon Press; 1995: $67 \mathrm{ff}$

47 Spiewak R. Immunotherapy of allergic contact dermatits. Immunotherapy 2011; 3: 979-996
48 Tarasov N. Die Blockflötenpräsenz auf der Musikmesse Frankfurt 2011. Windkanal - Das Forum für die Blockflöte 2011; 2: 20-24

49 Uccusic P. Bienenprodukte: Doktor Biene - Ihre Heilkraft und Anwendung in der Heilkunst. 1. Aufl.; München: Heyne; 1990

50 Vine K, De Leo V. Dermatologic manifestations of musicians: a case report and review of skin conditions in musicians. Cutis 2011; 87: $117-121$

51 Vieths $S$. Current understanding of cross-reactivity of food allergens and pollen. Ann N Y Acad Sci 2009; 964: 47-68

52 Vollbrink $U$. Flötenholz: Grenadill - Impressionen rund um einen edlen Rohstoff. Windkanal - Das Forum für die Blockflöte 2009; 2: $20 \mathrm{ff}$

53 White JM. Patch testing: What allergists should know. Clin Exp Allergy 2011; 42: 180 - 185

54 Wilson MS, Maizels RM. The innate immune system and its role in allergic disorders. Clin Rev Allergy Immunol 2004; 26: 35 -50 